\title{
Multiple Instance Learning Convolutional Neural Networks for Object Recognition
}

\author{
Miao Sun*, Tony X. Han*, Ming-Chang Liu ${ }^{\dagger}$ and Ahmad Khodayari-Rostamabad ${ }^{\dagger}$ \\ ${ }^{*}$ Electrical and Computer Engineering \\ University of Missouri, Columbia, Missouri 65211 \\ Email: msqz6@mail.missouri.edu, hantx@missouri.edu \\ †Sony Electronics Inc, San Jose, California, 95112 \\ Email: Ming-Chang.Liu, Ahmad.Khodayari@am.sony.com
}

\begin{abstract}
Convolutional Neural Networks (CNN) have demonstrated its successful applications in computer vision, speech recognition, and natural language processing. For object recognition, CNNs might be limited by its strict label requirement and an implicit assumption that images are supposed to be targetobject-dominated for optimal solutions. However, the labeling procedure, necessitating laying out the locations of target objects, is very tedious, making high-quality large-scale dataset prohibitively expensive. Data augmentation schemes are widely used when deep networks suffer the insufficient training data problem. All the images produced through data augmentation share the same label, which may be problematic since not all data augmentation methods are label-preserving. In this paper, we propose a weakly supervised CNN framework named Multiple Instance Learning Convolutional Neural Networks (MILCNN) to solve this problem. We apply MILCNN framework to object recognition and report state-of-the-art performance on three benchmark datasets: CIFAR10, CIFAR100 and ILSVRC2015 classification dataset.
\end{abstract}

\section{INTRODUCTION}

Deep learning algorithms [1] are revolutionizing various tasks in artificial intelligence including natural language processing $[2]-[5]$, speech recognition $[6]-[8]$, and computer vision [9]-[11]. The successes of deep learning algorithms are the result of its excellent capability to discover intricate structures in high-dimensional data with little manual engineering. The breakthroughs in ImageNet challenge [12] has demonstrated that powerful feature representations can be learned from data automatically, outdating traditional approaches based on hand-designed features.

The most successful algorithm in deep learning algorithms for image recognition is Convolutional Neural Networks $(\mathrm{CNN})[9],[13]-[15]$. The architecture of typical CNNs [16] is a stack of convolutional, non-linear, pooling and fullyconnected layers, followed by a loss function layer. It is designed to take advantages of local connections, shared weights, pooling and the use of many layers to learn highlevel representations of natural images, and it has demonstrated significant improvement over various benchmark object recognition datasets [12], [17], [18]. However, deep CNNs necessitate large well-labeled training data to achieve these superior results, whereas the labeling work is very tedious and expensive by hand. Limited amount or inferior quality of training data will lead to suboptimal models. Currently,

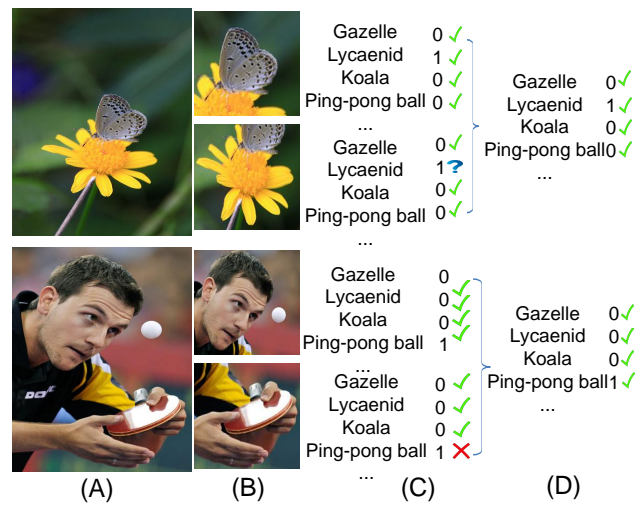

Fig. 1. Data augmentation and labels: (A) Original images from the ILSVRC2015 training set. (B) Image regions created for deep CNNs by data augmentation. (C) Labels assigned to created image areas. Check marks represent high-quality label. The question mark is a low quality label. Cross marks mean incorrect labels. (D) Image-level labels. All created regions share the same image-level label, which is intrinsically associated with multiple instance learning.

ImageNet, the largest labeled high-resolution image database available publicly, has around 14 million images and 22 thousand synsets. ILSVRC2015 is a subset of ImageNet with roughly 1,300 images in each of 1000 categories. In all, there are about 1.3 million training images, 50,000 validation images, and 150,000 testing images. For deep CNNs with million-level parameters, ILSVRC2015 is the most widely used dataset for training deep convolutional neural networks. Krizhevsky et. al [9] used a simple data augmentation scheme of generating image translations and horizontal reflections to increase the size of the 1.3 million training set by 2048 times. Without the scheme, deep networks would suffer substantial overfitting. Thus, this straightforward and powerful scheme is considered to be default for recent deep CNNs [13]-[15]. [19], [20] showed the promising performance could be achieved by high-quality bounding box proposals instead of randomly cropping images.

In practice, not all data augmentation methods are labelpreserving, especially when target objects fail to dominate the whole images. As a supervised learning method, $\mathrm{CNNs}$ take (data, label) pairs as in Fig 1 (B-C). The inferior quality 
labels make it difficult for optimization and lead to suboptimal $\mathrm{CNN}$ models. To reduce the effect of noisy training pairs, we model deep CNNs in a weakly supervised learning framework. Instead of assigning labels to all generated images (as in Fig 1 (C)), we treat the generated images as a bag and the original label as bag-level label (as in Fig 1(D)). This phenomenon is intuitively the problem of Multiple Instance Learning (MIL). For binary MIL, a bag is labeled positive if the bag contains at least one positive instance, and it is labeled negative if all its instances are negative. Therefore, incorporating MIL into deep learning algorithm would fully utilize the potential of the training set and achieve better performance.

Recently, $\mathrm{He}$ et al. [15] built a 152-layer deep network for high-resolution images [12] and over 1000-layer deep network for low-resolution images [17] by taking advantages of carefully designed initialization and residual learning techniques. The significant increase of depth of layers results in outperforming previous CNNs in various benchmark datasets [12], [18], [21].

In this paper, we propose a weakly supervised CNN framework named Multiple Instance Learning Convolutional Neural Networks (MILCNN) to reduce the effect of noisy labels. The proposed algorithms are validated by extensive experiments and have shown state-of-the-art performance on various object recognition benchmarks.

\section{MILCNN FOR IMAGE ClassifiCATION}

In this section, we will first briefly introduce mathematical formulations of CNNs including how to predict labels and update parameters. Then, we will give detailed equations about how to incorporate multiple instance learning into CNN. The whole procedure is summarized in Algorithm 1

\section{A. Traditional CNN Formulation}

Convolutional Neural Networks (CNN) are a special kind of neural networks, which consist a stack of convolutional layers, pooling layers, fully-connected layers and loss function layers. Optimization of CNNs is a supervised learning process to minimize loss function:

$$
L=\sum_{i=1}^{N} L_{i}=\sum_{i=1}^{N} f_{l o s s}\left(\mathbf{y}_{\mathbf{i}}, F\left(\mathbf{W}, \mathbf{x}_{\mathbf{i}}\right)\right)
$$

$L$ is the total cost of $N$ training examples. $\mathbf{y}_{\mathbf{i}}=\{0,1\}^{1 \times C}$ is the label matrix, where $C$ is the number of categories. W represents the collection of adjustable parameters in the structure. $\mathbf{x}_{\mathbf{i}} \in \Re^{1 \times D}$ is the input pattern matrix, where $D$ is the dimension of each input pattern. $F\left(\mathbf{W}, \mathbf{x}_{\mathbf{i}}\right) \in \Re^{1 \times C}$ can be interpreted as the category label of input patterns or probabilities associated with each category. $F$ indicates a set of functions in $\mathrm{CNN}$ such as convolutional functions, pooling functions and fully-connected functions. For notational convenience, we will drop the example index and use $\mathbf{x}$ to denote the chosen input data and $y$ the corresponding label. Then the Eq (1) will be

$$
L=f_{l o s s}(\mathbf{y}, F(\mathbf{W}, \mathbf{x}))
$$

The most widely used loss function in convolutional neural networks is softmax with cross-entropy loss function. Let CNN output $\mathbf{h}=F(\mathbf{W}, \mathbf{x}), \mathbf{h}=\left\{h_{1}, h_{2}, \ldots, h_{C}\right\}$, the predicted label is max value of $\mathbf{h}$

$$
\hat{y}=\operatorname{argmax}_{i=1}^{C}\left(h_{i}\right)
$$

and the cross-entropy loss is

$$
f_{\text {loss }}=-\sum_{i=1}^{C} y_{i} \log \left(p_{i}\right)
$$

where $p_{i}=\frac{\exp \left(h_{i}\right)}{\sum_{j=1}^{C} \exp \left(h_{j}\right)}$ for $i=1,2, \ldots C$

The gradient of softmax with cross-entropy loss w.r.t $\mathbf{h}$ is

$$
\frac{\partial f_{\text {loss }}}{\partial h_{i}}=-y_{i}+p_{i} \sum_{j=1}^{C} y_{j}
$$

According to chain rules, we will have

$$
\frac{\partial f_{l o s s}}{\partial \mathbf{W}}=\frac{\partial f_{\text {loss }}}{\partial \mathbf{h}} \frac{\partial \mathbf{h}}{\partial \mathbf{W}}
$$

The parameters of the $\mathrm{CNN}$ is updated as

$$
\mathbf{W}_{\text {new }}=\mathbf{W}_{\text {old }}-\lambda \frac{\partial f_{\text {loss }}}{\partial \mathbf{W}}
$$

In the simplest case, the learning rates $\lambda$ is a scalar constant.

1) MILCNN Formulation: For multiple instance learning, training instances are not singletons. Instead, they come in "bags", where all the examples in a bag share the same label. For object recognition, regions in each image are considered as a bag as in Fig 1(B).

Let $B=\left\{\mathbf{x}^{1}, \mathbf{x}^{2} \ldots \mathbf{x}^{m}\right\}, \mathbf{x}^{m}$ is $m_{\text {th }}$ region in the image. The loss function w.r.t to the bag $B$ is

$$
f_{\text {loss }}=-\sum_{i=1}^{C} y_{i} \log \left(p\left(c_{i}=1 \mid B\right)\right)
$$

where $p\left(c_{i}=1 \mid B\right)$ represents the probability that the bag is classified into $i_{t h}$ category. According to concept of multiple instance learning, $B$ is a negative bag for the $i_{t h}$ category if all the instances in the bag are negative:

$$
p\left(c_{i}=0 \mid B\right)=\Pi_{j=1}^{m}\left(1-p\left(c_{i}=1 \mid \mathbf{x}^{j}\right)\right)
$$

$p\left(c_{i}=1 \mid \mathbf{x}^{j}\right)$ is the probability of $j_{t h}$ region to be considered as $i_{t h}$ category, and

$$
p\left(c_{i}=1 \mid \mathbf{x}^{j}\right)=1-\exp \left(-\lambda h_{i}^{j}\right)
$$

where $h_{i}^{j}$ is the $i_{t h}$ output of CNN model before loss layer for $j_{t h}$ region , and $h_{i}^{j} \in[0, \infty$ ) (if there is a ReLU layer before the loss function layer), $\lambda$ is constant positive value. The reason why we define the probability as Eq 10 is to simplify calculation of gradients below. 
Optimization of Eq (8) is equivalent to minimization of the following function

$$
f_{\text {loss }}=-\sum_{i=1}^{C}\left(1-y_{i}\right) \log \left(1-p\left(c_{i}=1 \mid B\right)\right)
$$

Combining Eq (11) with Eq (10, 9) will have

$$
\begin{aligned}
f_{\text {loss }} & =-\sum_{i=1}^{C}\left(1-y_{i}\right) \log \left(1-p\left(c_{i}=1 \mid B\right)\right) \\
& =-\sum_{i=1}^{C}\left(1-y_{i}\right) \log \left(p\left(c_{i}=0 \mid B\right)\right) \\
& =-\sum_{i=1}^{C}\left(1-y_{i}\right) \log \Pi_{j=1}^{m}\left(1-p\left(c_{i}=1 \mid \mathbf{x}^{j}\right)\right) \\
& =-\sum_{i=1}^{C}\left(1-y_{i}\right) \sum_{j=1}^{m} \log \left(1-p\left(c_{i}=1 \mid \mathbf{x}^{j}\right)\right) \\
& =-\sum_{i=1}^{C}\left(1-y_{i}\right) \sum_{j=1}^{m} \log \left(\exp \left(-\lambda h_{i}^{j}\right)\right) \\
& =-\sum_{i=1}^{C}\left(1-y_{i}\right) \sum_{j=1}^{m}\left(-\lambda h_{i}^{j}\right)
\end{aligned}
$$

Then the corresponding gradients will be

$$
\frac{\partial f_{\text {loss }}}{\partial h_{i}^{j}}=\lambda\left(1-y_{i}\right)
$$

Given all the equations above, MILCNN can be summarized in Algorithm 1

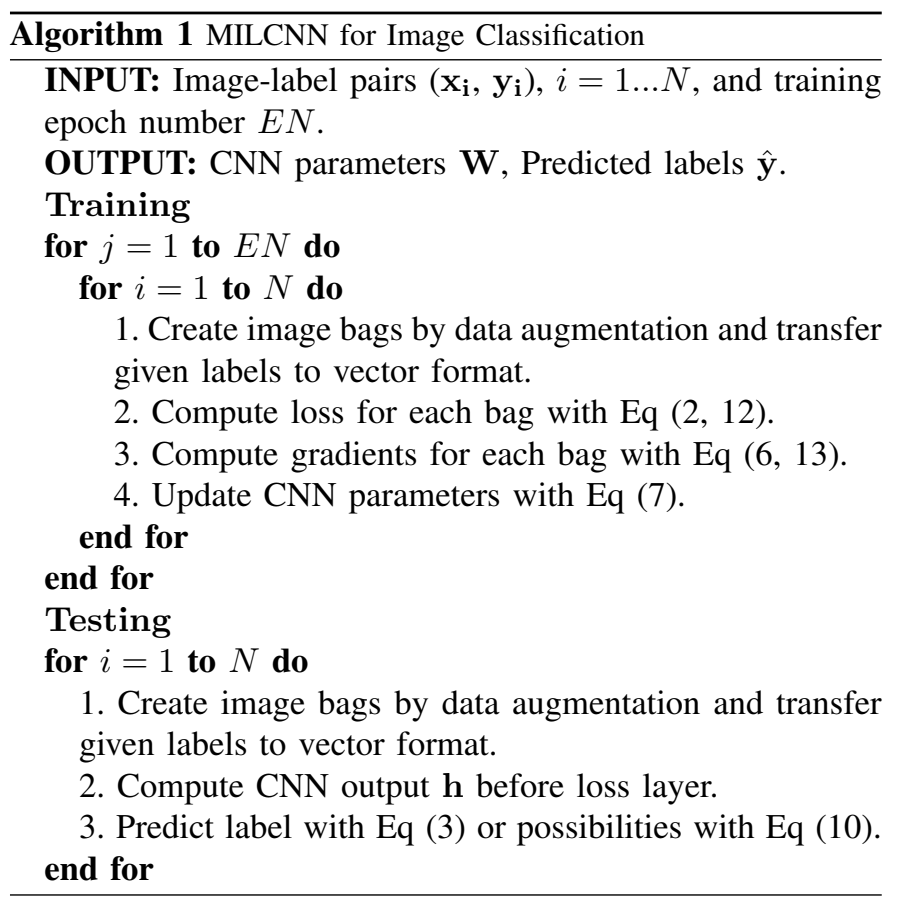

Discussion of predicted possibilities: In the conventional setup, the output of softmax layer is considered as predicted possibilities. However, softmax layer is only suitable for single label problem, which means the input image only contains one target object. For example, if one image $\mathbf{x}$ has target objects, such as "Ping-Pong ball" and "Person", it will confuse the model because $\max _{i=1}^{C} p_{i}$ is trying to reach 0.5 instead of 1 for the ground truth category. Therefore, we discard the softmax layer and use Eq 10 in the MILCNN framework instead. Eq (10) indicates that there are chances that input images can contain multiple target objects or contain no target objects at all. Compared to softmax function, the possibility is not based on one input image but related to all training images.

\section{EXPERIMENTS}

In this section, we will first talk about detailed configurations of MILCNN structures, and then we will provide state-of-the-art performance on benchmark datasets: CIFAR10, CIFAR100 and ILSVRC2015 classification dataset.

\section{A. MILCNN Structures}

Our MILCNN is a combination of deep residual network [15] and multiple instance learning loss layer as in Fig 2 MILCNN is a stack of layers including convolutional layers, batch normalization layers [22], rectified linear unit (ReLU) [23], residual network layers [15], pooling layers, fully-connected layers and multiple instance learning loss function, which is developed in Section II-A1 1

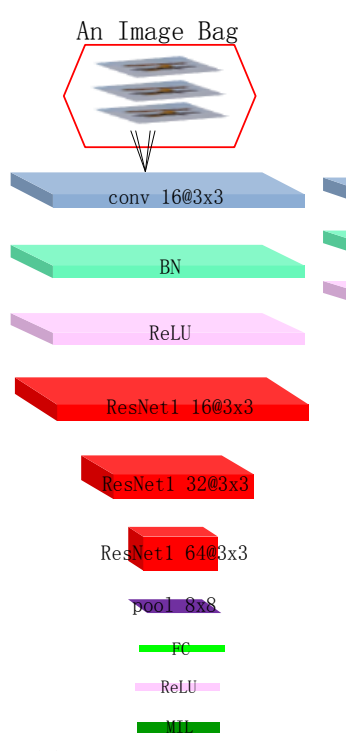

(A) MILCNN for CIFAR10

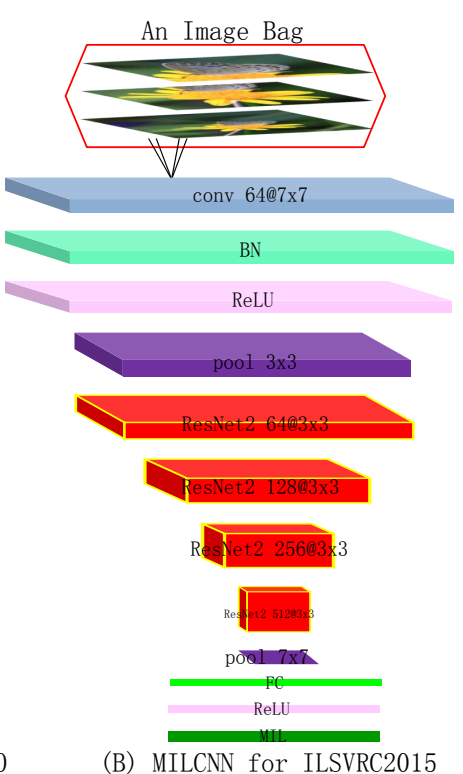

(B) MILCNN for ILSVRC2015
Fig. 2. MILCNN Structures: Use images bags for both structures. conv $16 @ 3 \times 3$ means there are 16 convolutional kernels with receptive field size 3 by 3 . Two different residual network blocks are used for two datasets separately and details in residual network blocks are illustrated in Fig 3

Convolutional layers make use of local connections and shared weights, which is effective to avoid overfitting compared to fully-connected layers. Thus, we only use one fullyconnected layer to project feature maps to the number of

\footnotetext{
${ }^{1}$ The detailed residual networks are based on the work of Sam Gross and
} Micheal Wiber called "Training and investigating Residual Nets". 


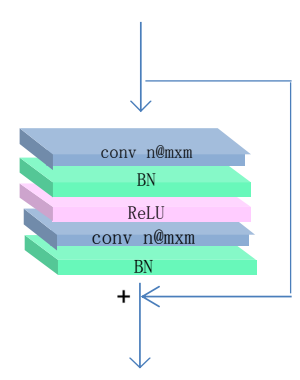

(A) ResNet1

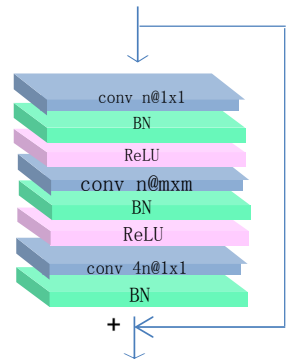

(B) ResNet2
Fig. 3. Residual Network Blocks: Two different residual network block types used in Fig 2 conv $n @ m \times m$ means $n$ convolutional kernel with size $m$ by $m$.

categories of a training set. Batch Normalization has been verified to be able to accelerate deep networks training via reducing covariate shift. ReLU is used to replace traditional nonlinearity such as sigmoid and tangent functions, which tend to slow down learning procedure due to saturating effect. Multiple instance learning loss layer is developed in this paper to work harmony with data augmentation to fully explore the potential of training sets.

With network depth increasing, accuracy gets saturated and then degrades rapidly, that is, degradation problem. Degradation is not caused by overfitting but related to the difficulty of optimization of substantial deep neural networks. This issue is recently addressed by residual networks. [15] shows it is much easier to optimize the residual mapping with reference mapping than to optimize the original, unreferenced mapping. Figure 3 shows two implementations of residual networks using shortcut connections. Shortcut connections are those skipping one or more layers, and identity mapping is most widely used as shortcut connections. The stacked nonlinear layers in Fig 3 is therefore called residual mapping.

\section{B. CIFAR10}

CIFAR10 [17] is a dataset of RGB images containing 32 x 32 pixels. It has 10 categories with 50,000 training images and 10,000 test images.

Our best MILCNN structure for CIFAR10 is in Fig 2 (A) and detailed layer setup is summarized in Table I. Configurations of residual network blocks are shown in the brackets. In the bracket, two $3 \times 3,16$ represents 16 convolutional kernels with receptive field size 3 by 3 for Type 1 residual network block in Fig 3.A). For each convolutional layer, we choose stride 1 and padding size 1 so that the output feature map will not change. The downsampling is only performed by first convolutional layer in ResNet2 and ResNet3 with a stride of 2 , so the output size will decrease by a factor of 2 . Batch normalization layer and ReLU layer do not affect sizes of feature map and thus they are not listed in this table. We refer this configuration as 111-layer following convection of [15] by excluding batch normalization layers, ReLU layers and loss layer. If taking all these layers into consideration, the total number of layers will be $1+1+1+5 \times 18 \times 3+1+1+1+1=$ 277.

TABLE I

CNN ARCHITECTURE FOR CIFAR10.

\begin{tabular}{c|c|c}
\hline layer name & output size & 111-layer \\
\hline conv1 & $32 \times 32$ & $3 \times 3,16$, stride 1, padding 1 \\
\hline ResNet1 & $32 \times 32$ & {$\left[\begin{array}{l}3 \times 3,16 \\
3 \times 3,16\end{array}\right] \times 18$} \\
\hline ResNet2 & $16 \times 16$ & {$\left[\begin{array}{l}3 \times 3,32 \\
3 \times 3,32\end{array}\right] \times 18$} \\
\hline ResNet3 & $8 \times 8$ & {$\left[\begin{array}{l}3 \times 3,64 \\
3 \times 3,64\end{array}\right] \times 18$} \\
\hline pool2 & $1 \times 1$ & average pool $8 \times 8$ \\
\hline fc & 10 & fc size $64 \times 10$
\end{tabular}

During training of this MILCNN, we use a weight decay of 0.0001 and momentum 0.9 . The initial learning rate is 0.1 and reduces to $0.01,0.001$ at 80 epochs and 120 epochs. We form image bags by sampling $532 \times 32$ regions from a 4 pixel padded images, that is, the image resolution is $40 \times 40$. The best performance is $5.1 \%$ error rate and achieves superior results compared to other state-of-the-art methods as shown in Table [I]

TABLE II

TEST SET ERROR RATES FOR CIFAR-10 OF VARIOUS METHODS

\begin{tabular}{lc}
\hline METHOD & TEST ERROR \\
\hline CONV. MAXOUT [24] & $9.38 \%$ \\
DroPCONNECT +12 NETWORKS [25] & $9.32 \%$ \\
NIN [26] & $8.81 \%$ \\
RESNET(110-LAYER) [15] & $6.43 \%$ \\
\hline \hline RESNET (111-LAYER) & $6.07 \%$ \\
MILCNN (111-LAYER) & $5.11 \%$
\end{tabular}

\section{CIFARIO0}

CIFAR100 [17] is a dataset of RGB images containing 32 $\mathrm{x} 32$ pixels. It has 100 categories with 50,000 training images and 10,000 test images.

Our best MILCNN structure for CIFAR100 is similar to Fig 2 (A) and Table I, except that the last fully-connected layer has 100 output nodes. The training hyper-parameters and procedure are also similar to Secion III-B

In Table III. ResNet (111-layer) has achieved the state-ofthe-art performance with the help of residual network blocks. However, after incorporating our multiple instance learning loss layer, the performance has a significant improvement to $26.42 \%$ for a single model.

\section{ILSVRC2015}

ILSVRC2015 is a subset of ImageNet classification dataset, and it contains 1.28 million training images and 50,000 validation images. 
TABLE III

TEST SET ERROR RATES FOR CIFAR-100 OF VARIOUS METHODS

\begin{tabular}{lc} 
METHOD & TEST ERROR \\
\hline LEARNED POOLING [27] & $43.71 \%$ \\
STOCHASTIC POOLING [28] & $42.51 \%$ \\
CONV. MAXOUT [24] & $38.57 \%$ \\
TREE BASED PRIORS [29] & $36.85 \%$ \\
NIN [26] & $35.68 \%$ \\
\hline RESNET (111-LAYER) & $28.54 \%$ \\
MILCNN (111-LAYER) & $26.42 \%$
\end{tabular}

Our best MILCNN structure for ILSVRC2015 is in Fig 2 (B) and detailed layer setup is summarized in Table IV. Compared to configurations in Fig 2 (A), we add one pooling layer after the first convolutional layer and choose the second type residual network block in Fig 3 These modifications are mainly due to high-resolution images, whose resolution is 224 by 224 (The original resolution of images in ILSVRC2015 classification dataset is around 500 by 400, we resize and crop $224 \times 224$ for our $\mathrm{CNN}$ architecture input). In the brackets, convolutional kernels with receptive field size $1 \times 1$ are adopted to reduce parameters of CNN models. For shortcut connections, type 2 residual network blocks do not use identity mapping but a project matrix implemented via $1 \times 1$ convolutions to match dimensions. The downsampling is only performed by middle convolutional layer in ResNet2, ResNet3 and ResNet4 with a stride of 2 , so the output feature map will decrease by a factor of 2 . We refer this configuration as 103-layer following convection of $[15]$ by excluding batch normalization layers, ReLU layers and loss layer. If taking all these layers into consideration, the total number of layers will be $1+1+1+1+8 \times(3+4+23+3)+1+1+1+1=272$.

TABLE IV

CNN ARCHITECTURE FOR ILSVRC2015.

\begin{tabular}{c|c|c}
\hline layer name & output size & 103 -layer \\
\hline conv1 & $112 \times 112$ & $7 \times 7,64$, stride 2 , padding 3 \\
\hline pool1 & $56 \times 56$ & $3 \times 3$, stride 2 , padding 1 \\
\hline ResNet1 & $56 \times 56$ & {$\left[\begin{array}{c}1 \times 1,64 \\
3 \times 3,64 \\
1 \times 1,256\end{array}\right] \times 3$} \\
\hline ResNet2 & $28 \times 28$ & {$\left[\begin{array}{c}1 \times 1,128 \\
3 \times 3,128 \\
1 \times 1,512\end{array}\right] \times 4$} \\
\hline ResNet3 & $14 \times 14$ & {$\left[\begin{array}{c}1 \times 1,256 \\
3 \times 3,256 \\
1 \times 1,1024\end{array}\right] \times 23$} \\
\hline ResNet4 & $7 \times 7$ & {$\left[\begin{array}{c}1 \times 1,512 \\
3 \times 3,512 \\
1 \times 1,2048\end{array}\right] \times 3$} \\
\hline pool2 & $1 \times 1$ & average pool $7 \times 7$ \\
\hline fc & 1000 & fc size $2048 \times 1000$ \\
\hline
\end{tabular}

During training of this MILCNN, we notice that multiple instance learning loss function is slower than softmax with entropy loss function. So instead of training MILCNN from scratch, we use Softmax with entropy loss function for pretraining. In details, we set a weight decay as 0.0001 and momentum as 0.9. The initial learning rate is 0.1 and reduces to $0.01,0.001$ at 30 epochs and 60 epochs. We choose same weights initialization method in [30]. The best model has achieved $21.08 \%$ top 1 error rate with the aforementioned configurations. Then we switch to our designed multiple instance learning loss function and $\lambda$ is set 0.001 . We form image bags by sampling $5224 \times 224$ regions from images of resolution $256 \times 256$. Compared to the original deep residual networks [15], where the best performance is achieved at 152layer deep network, our structure only contains 103 layers due to the limitation of GPU memories (It will take about $11 \mathrm{G}$ memory for batch size 64).

TABLE V

TEST SET ERROR RATES FOR ILSVRC2015 OF VARIOUS METHODS

\begin{tabular}{lc|c}
\hline METHOD & TOP-1 ERROR & TOP-5 ERROR \\
\hline VGG [31] (ILSVRC'14) & - & 8.43 \\
GOOGLENET [13] (ILSVRC'14) & - & 7.89 \\
\hline VGG [31] (V5) & 24.4 & 7.1 \\
PRELU-NET [30] & 21.59 & 5.71 \\
BN-INCEPTION [22] & 21.99 & 5.81 \\
RESNET(101-LAYER) [15] & 19.87 & 4.60 \\
RESNET(152-LAYER) [15] & 19.38 & 4.49 \\
\hline \hline RESNET(103-LAYER) & 21.08 & 5.35 \\
MILCNN(103-LAYER) & 20.78 & 5.30
\end{tabular}

From Table V, our implementation of residual networks has achieved the state-of-the-art performance compared to other methods for single model evaluation on validation dataset. Our single MILCNN model is able to further improve the performance to $5.30 \%$ and it is reaching the human-level performance $5.10 \%$ [32].

Fig 4 shows some predictions of validation images. We randomly select some categories and each category has two images: top one is successfully classified with top 1 predicted labels, bottom one is incorrectly classified with top 1 predicted labels. Since MILCNN still choose relatively large regions to form bags, our algorithm still makes mistakes in cases that have relatively small objects. For example, both images in the eighth column are in "Pitcher" category, but the bottom "Pitcher" is too small to be visible. MILCNN can also make less mistakes when more context information is considered, such as the images in the seventh row. The bottom image is labeled as "Matchstick" while the ground truth is "Megalith". If taking the context information(positions and scale of person in the image), MILCNN is supposed to rule out the possibility of "Matchstick" at least.

\section{CONClusion}

We provided a weakly supervised framework for image recognition by combining multiple instance learning loss and deep residual networks. We presented mathematical formulation for how to incorporate the concept of multiple instance learning to a deep learning architecture. Besides, we showed state-of-the-art performance on both low-resolution 


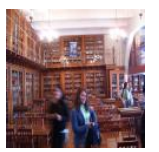

Library

Bookshop

Tobacco shop

Bookcase

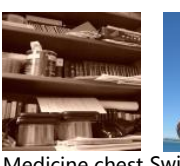

Medicine ches

Bookcase

Hair spry

Wine bottle
Bookshop

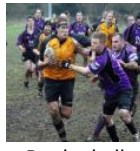

Rugby ball

Soccer ball

Basketball

ootball helmet

Parachute

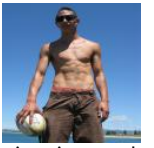

Sarong

Volleyball

Bathing cap

Sunscreen

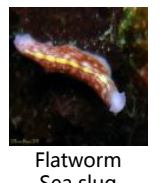

Sea slug

Eel

Slug

Conch

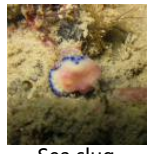

Sea slug

Flatworm

Coral reef

Eel
Eel

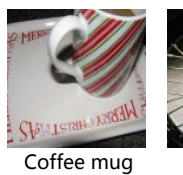

Espresso

Cup

Teapot

Coffeepot

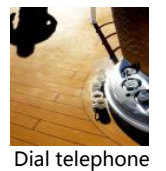

Dial telephone

Disk brak

Reel

Car wheel
Joystick
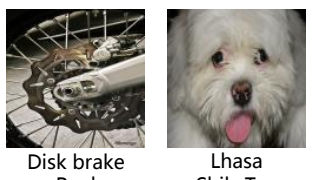

Shih-Tzu

Toy poodle

Miniature poodle

Irish terrier

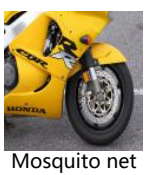

Disk brake

Car wheel

Moped

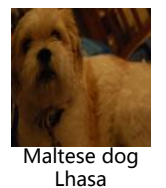

Lhasa

Shih-Tzu

Tibetan terrie
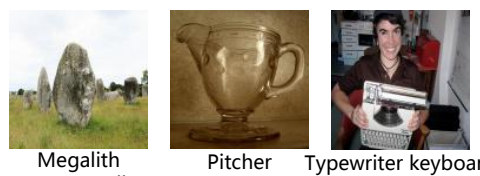

Pitcher Typewriter keyboard Chimpanze

Cup Space bar Orangutan

Coffeepot Joystick Siamang

Coffee mug Computer keyboard Gorilla

Water jug Sewing machine Gibbon

Fig. 4. Visualization: First row are examples validation images successfully classified with top 1 predicted labels. Second row are examples with incorrect top 1 predicted labels. Each column shares same ground-truth label.

CIFAR datasets and high-resolution ILSVRC2015 classification dataset.

\section{ACKNOWLEDGMENT}

The authors would like to thank Sony Electronics Inc. for their generous funding of this work.

\section{REFERENCES}

[1] Y. LeCun, Y. Bengio, and G. Hinton, "Deep learning," in Nature, vol. 521,2015 , pp. 436-444.

[2] R. Collobert, J. Weston, L. Bottou, M. Karlen, K. Kavukcuoglu, and P. Kuksa, "Natural language processing (almost) from scratch," in arXiv:1103.0398, 2011.

[3] A. Bordes, S. Chopra, and J. Weston, "Question answering with subgraph embeddings," in arXiv:1406.3676, 2014.

[4] S. Jean, K. Cho, R. Memisevic, and Y. Bengio, "On using very large target vocabulary for neural machine translation," in arXiv:1412.2007, 2015.

[5] I. Sutskever, O. Vinyals, and Q. V. Le, "Sequence to sequence learning with neural networks," in arXiv:1409.3215, 2014.

[6] T. Mikolov, A. Deoras, D. Povey, L. Burget, and J. H. Cernocky, "Strategies for training large scale neural network language models," in IEEE Automatic Speech Recognition and Understanding Workshop, 2011.

[7] G. Hinton, L. Deng, D. Yu, A. Mohamed, N. Jaitly, A. Senior, V. Vanhoucke, P. Nguyen, T. S. G. Dahl, and B. Kingsbury, "Deep neural networks for acoustic modeling in speech recognition," in IEEE Signal Processing Magazine 29, pp. 82-97, 2012.

[8] T. Sainath, A. Mohamed, B. Kingsbury, and B. Ramabhadran, "Deep convolutional neural networks for lvcsr," in Proc. Acoustics, Speech and Signal Processing, pp. 8614-8618, 2013.

[9] A. Krizhevsky, I. Sutskever, and G. E. Hinton, "Imagenet classification with deep convolutional neural networks," in Neural Information Processing Systems, NIPS 2012

[10] C. Farabet, C. Couprie, L. Najman, and Y. LeCun, "Learning hierarchical features for scene labeling," in IEEE Transactions on Pattern Analysis and Machine Intelligence, 2013 vol. 35, no. 8, pp. 1915-1929.

[11] J. Tompson, A. Jain, Y. LeCun, and C. Bregler, "Joint training of a convolutional network and a graphical model for human pose estimation," in arXiv: 1406.2984, 2014.

[12] J. Deng, A. Berg, S. Satheesh, H. Su, A. Khosla, and L. FeiFei., "Imagenet large scale visual recognition competition 2012 (ilsvrc2012)," 2012. [Online]. Available: http://www.image-net.org/ challenges/LSVRC/2012/.
[13] C. Szegedy, W. Liu, Y. Jia, P. Sermanet, S. Reed, D. Anguelov, D. Erhan, V. Vanhoucke, and A. Rabinovich, "Going deeper with convolutions," in arXiv:1409.4842, 2014

[14] S. Ren, K. He, R. Girshick, and J. Sun, "Faster r-cnn: Towards real-time object detection with region proposal networks," in arXiv:1506.01497, 2016.

[15] K. He, X. Zhang, S. Ren, and J. Sun, "Deep residual learning for image recognition," in arXiv:1512.03385, 2015.

[16] Y. LeCun, L. Bottou, Y. Bengio, and P. Haffner., "Gradient-based learning applied to document recognition," in Proc. of the IEEE, 1998

[17] A. Krizhevsky, "Learning multiple layers of features from tiny images," in MSc thesis, 2009.

[18] M. Everingham, L. Van Gool, C. K. I. Williams, J. Winn, and A. Zisserman, "The PASCAL Visual Object Classes Challenge 2007 (VOC2007) Results," http://www.pascalnetwork.org/challenges/VOC/voc2007/workshop/index.html, 2007.

[19] Y. Wei, W. Xia, J. Huang, B. Ni, J. Dong, Y. Zhao, and S. Yan, "Cnn: Single-label to multi-label," in arXiv:1406.5726, 2014.

[20] M. Sun, T. X. Han, and X. Xu, "Latent model ensemble with autolocalization," in arXiv:1604.04333, 2016

[21] T.-Y. Lin, M. Maire, S. Belongie, L. Bourdev, R. Girshick, J. Hays, P. Perona, D. Ramanan, C. L. Zitnick, and P. Dollr, "Microsoft coco: Common objects in context," in arXiv:1405.0312, 2015.

[22] S. Ioffe and C. Szegedy, "Batch normalization: Accelerating deep network training by reducing internal covariate shift," in arXiv:1502.03167, 2015.

[23] V. Nair and G. Hinton, "Rectified linear units improve restricted boltzmann machines," in ICML, 2010.

[24] I. J. Goodfellow, D. Warde-Farley, M. Mirza, A. Courville, and Y. Bengio, "Maxout networks," in arXiv:1302.4389, 2013.

[25] L. Wan, M. Zeiler, S. Zhang, Y. L. Cun, and R. Fergus., "Regularization of neural networks using dropconnect," in ICML, 2013.

[26] M. Lin, Q. Chen, and S. Yan, "Network in network," in ICLR, 2014

[27] M. Malinowski and M. Fritz., "Learnable pooling regions for image classification." in arXiv:1301.3516, 2013.

[28] M. D. Zeiler and R. Fergus., "stochastic pooling for regularization of deep convolutional neural networks," in arXiv:1301.3557, 2013.

[29] N. Srivastava and R. Salakhutdinov., "Discriminative transfer learning with tree-based priors," in Neural Information Processing Systems, 2013.

[30] K. He, X. Zhang, S. Ren, and J. Sun, "Delving deep into rectifiers: Surpassing human-level performance on classification." in ICCV, 2015

[31] K. Simonyan and A. Zisserman, "Very deep convolutional networks for large-scale image recognition," in arXiv:1409.1556, 2015.

[32] O. Russakovsky, J. Deng, H. Su, J. Krause, S. Satheesh, S. Ma Z. Huang, A. Karpathy, A. Khosla, M. Bernstein, A. C. Berg, and L. Fei-Fei, "Imagenet large scale visual recognition challenge," in arXiv:1409.0575, 2014. 